\title{
Benign paroxysmal positional vertigo in patients after mild traumatic brain injury
}

\author{
Magdalena Józefowicz-Korczyńska ${ }^{1, A, C-F}$, Anna Pajor ${ }^{1, C-E}$, Wojciech Skóra ${ }^{2, B-D}$ \\ ${ }^{1}$ Department of Otolaryngology, Medical University of Lodz, Poland \\ 2 Otolaryngology Department, The Stanisław Rybicki Memorial Regional Hospital, Skierniewice, Poland \\ A - research concept and design; $\mathrm{B}$ - collection and/or assembly of data; $\mathrm{C}$ - data analysis and interpretation; \\ $D$ - writing the article; $E$ - critical revision of the article; $F$ - final approval of the article
}

Address for correspondence

Magdalena Józefowicz-Korczyńska

E-mail:magdalena.jozefowicz-korczynska@umed.lodz.pl

\section{Funding sources}

A grant from the Medical University of Lodz (No. 503/2-036-02/503-21-002).

\section{Conflict of interest}

None declared

Received on December 5, 2016

Reviewed on February 9, 2017

Accepted on March 23, 2017
DOI

10.17219/acem/69708

\section{Copyright}

Copyright by Author(s)

This is an article distributed under the terms of the

Creative Commons Attribution Non-Commercial License

(http://creativecommons.org/licenses/by-nc-nd/4.0/)

\section{Abstract}

Background. Post-traumatic vertigo, dizziness and balance disorders following head trauma range from $15 \%$ to $78 \%$ in the general population. Benign paroxysmal positional vertigo (BPPV) is the most common vestibular disorder in such patients.

Objectives. The aim of the study was to assess the occurrence of BPPV in patients with mild traumatic brain injury (MTBI) and determine the outcome of treatment.

Material and methods. A group of 179 patients, with a mean age of 45.2 years, complaining of vertigo/ /dizziness and balance instability after MTBI, was enrolled into the study. All these patients were diagnosed and treated in the Department of Otolaryngology (Medical University of Lodz, Poland) between the years 2012 and 2014. Anamnesis and otoneurological examination were conducted in each patient. The diagnosis was based on the medical history, the Dix-Hallpike test or the rollover test. The treatment comprised the Epley, barbecue and particle repositioning (RM) maneuvers.

Results. Nineteen patients (10.6\%) complained about attacks of vertigo elicited by positional changes. The diagnosis of BPPV was confirmed in 9 (47.4\%) patients: 8 cases with a positive Dix-Hallpike test and 1 with the roll test. In 10 cases, a high probability of BPPV was diagnosed based on the medical history. Eight patients were treated by a single Epley maneuver and 1 patient by the barbecue roll. In 4 (44.4\%) patients, the maneuvers were repeated. On the follow-up examination, the patients were not found to have vertigo.

Conclusions. Benign paroxysmal positional vertigo should be diagnosed and treated successfully in patients after head trauma.

Key words: rehabilitation, benign paroxysmal positional vertigo, mild traumatic brain injury 


\section{Introduction}

Traumatic brain injury (TBI) is increasingly becoming more commonplace, with one of the most common subtypes being mild traumatic brain injury (MTBI). ${ }^{1-4}$ Although patients with severe TBI are more likely to seek medical attention at the outpatient ward, many patients with MTBI report to general practitioners or primary healthcare units at varying times after injury, or fail to report it at all. ${ }^{5}$ The diagnostic criteria for MTBI used by the American Congress of Rehabilitation Medicine comprise loss of consciousness, the presence of headaches, dizziness or changes in mental status, feeling dazed, disorientated or confused, and experiencing persistent post-traumatic amnesia, as well as cognitive and/or behavioral/emotional symptoms following the injury. ${ }^{6}$

In literature, the incidence of post-traumatic vertigo, dizziness and balance disorders following MTBI is estimated to range from $15 \%$ to $78 \%$ in the general population and to be approx. $14 \%$ in hospitalized patients. Although traumatic disequilibrium or vertigo is not a clinical entity, it refers to the etiology for the heterogeneous presentation of peripheral or central vestibular system dysfunction. The duration of post-traumatic balance system disturbances varies. While $53 \%$ of patients report dizziness occurring within 1 week after MTBI, $18 \%$ report symptoms persisting for at least 2 years. Post-traumatic balance dysfunction signs may occur in $10-15 \%$ of patients experiencing head trauma for 1 year or longer, and in $28 \%$ of those classified as MTBI. ${ }^{4,7}$

Benign paroxysmal positional vertigo (BPPV) is a disorder of the inner ear characterized by sudden episodes of severe rotatory vertigo triggered by rapid changes in head position relative to gravity. The provoked situations are lying down, tipping the head up or down, sitting up from a supine position, particularly after awaking in the morning, or turning over in bed. In addition, a reaction can be elicited by the patient looking upward, for example, to place an object on a shelf above the head, or bending forward to tie shoelaces. The majority of patients experience 1 or more seconds of latency following the movement before experiencing violent attacks of vertigo lasting 10-30 s and linear-rotatory nystagmus: the fast phase beating toward the undermost ear, or upward when the gaze is directed to the uppermost ear. ${ }^{7-9}$

A population-based study estimated BPPV to have a lifetime prevalence of $2.4 \%$ and accounts for $8 \%$ of individuals with moderate to severe dizziness and vertigo. ${ }^{10}$ The severity and frequency of the occurrence of BPPV differ and are unpredictable. Most BPPV sufferers experience the "benign" form, where the symptoms resolve spontaneously within weeks or months; however, the complains persist in $20-30 \%$ of untreated patients, recurring over a period of years in about $30 \%$ of cases. ${ }^{7,8,11}$

Benign paroxysmal positional vertigo is thought to be caused by micro-sized calcium crystals called otoconia, which become dislodged from their normal location in the utricle, one of the sensory organs of the inner ear. These detached otoconia may aggregate into larger clumps in the fluid-filled spaces of the inner ear. Since they are heavier than the endolymph, in $85-95 \%$ of cases, they become trapped in the most dependent part, the posterior semicircular canal, when the patient is in a supine position. This causes inertial changes in the endolymph and unwanted fluid flow in the semicircular canal, resulting in ampullofugal cupula deflection, whenever head motion is along the plane of the canal. In 5-20\% of cases, this debris could be displaced into the horizontal canal or, in $2-3 \%$ of cases, into the anterior canal. ${ }^{8,9}$

In several publications based on large numbers of BBPV cases, the cause of otoconial detachment could not be established in 34-66\% of idiopathic or primary BPPV cases, such as in elderly patients. ${ }^{7,8,12}$ Although some scanning electron microscopy studies have found otoconia to have degenerated, more than 1 explanation of BPPV is possible, even in elderly patients. ${ }^{7,8,11}$ The most common causes of acquired or secondary form of BPPV are thought to be head and labyrinth trauma, inner ear diseases such as viral neurolabyrinthitis or vestibular neuritis, Meniere's disease, vestibulobasilar ischemia and anterior inferior cerebellar artery (AICA) syndrome, or prolonged bedrest due to unrelated disease. Occasionally BPPV is experienced following general surgery or procedures on the inner ear, such as stapes surgery.,8

Many authors found that BPPV was the most common vestibular disorder in patients after head trauma. ${ }^{12-22}$ The onset typically appears just after trauma, or a few days or weeks after MTBI, and can be unilateral or bilateral. Even mild head injury could result in the detachment of otoconia in the endolymph. Post-traumatic development of BPPV can also occur as the result of a degenerative process following labyrinth concussion, where after some time, an otoconial clump will accumulate, which could flow to an inappropriate site in the inner ear. ${ }^{8}$

A diagnosis of posterior semicircular canal BPPV can be established by the Dix-Hallpike positional test. The patient, sitting on a couch, is abruptly tilted with the head turned and hanging in the lateral lying position. This elicits a typical attack of vertigo and nystagmus mentioned above. The nystagmus is best seen in Frenzel glasses. The signs and symptoms of BPPV commonly come and go, and episodes of vertigo can disappear for some time and then recur. Effective methods for treating posterior canal BPPV are the Epley and Semont repositioning maneuvers, which are designed to move the endolymphatic debris from the posterior semicircular canal into the vestibule. For horizontal BPPV, roll maneuvers such as the Lampert maneuver or barbecue roll are the most commonly employed..$^{23-25}$

The aim of the study was to assess the occurrence of BPPV in patients with MTBI and determine the outcome of treatment. 


\section{Material and methods}

A group of 179 patients complaining of vertigo/dizziness and balance instability after MTBI were enrolled into the study. All were diagnosed and treated in the Department of Otolaryngology (Medical University of Lodz, Poland), between the years 2012 and 2014. The study group comprised 98 women and 81 men, with a mean age of 45.2 ( \pm 11.8 years). The inclusion criteria were as follows: MTBI with or without short loss of consciousness according to the recommendations for MTBI diagnosis, an absence of meningeal and pathological focal signs in clinical neurological examination, and an absence of organic lesions under radiological imaging examination. ${ }^{6}$ All participants were fully informed about the aim of the study and the test procedure, and gave their informed consent to their participation. The study design was approved by the Ethics Committee (No. RNN/87/12KE).

The $1^{\text {st }}$ examination took place from 14 to 30 days following head trauma. Anamnesis and otoneurological examination were conducted in each patient. The diagnosis was based on a review of a medical history and the presence of nystagmus induced by the Hallpike-Dix test, confirming posterior semicircular canal BPPV, or the rollover test, for lateral canal BPPV, as given in consensus document of the Committee for the Classification of Vestibular Disorders of the Bárány Society. ${ }^{26}$

The balance system was evaluated by videonystagmography (VNG) (Ulmer SYNAPSYS 2008, Marseille, France), which recorded spontaneous ocular movements with eyes open and closed, as well as positional tests and a number of ocular-motor tests including the smooth pursuit, optokinetic and saccadic tests. Kinetic stimulation was also evaluated with the torsion swing test and the FitzgeraldHallpike caloric test.

The treatment comprised the Epley, barbecue and particle repositioning (RM) maneuvers. The Epley maneuver was performed specifically in the case of posterior semicircular canal (PSC) BPPV. ${ }^{25,27}$ In the Epley maneuver, the patient sat on the examination table and the examiner performed the Dix-Hallpike test to the affected side, which elicited the typical nystagmus and vertigo. This position was maintained for 1-2 min after the nystagmus subsided. The patient's head was then rotated $90^{\circ}$ toward the opposite side and held in this position for $30 \mathrm{~s}$.

The barbecue maneuver was performed specifically in the case of horizontal canal BPPV. Beginning in the supine position, the patient was asked to roll $90^{\circ}$ towards the unaffected side, and then turn the head $90^{\circ}$ to the unaffected side, thus positioning the head at an angle of almost $90^{\circ}$ to the body. This nose-down position was maintained for another 1-2 min, after which the patient was helped to sit up.

The symptoms occurring during each RM and at the end of the treatment session were recorded. The patients were instructed to avoid bending over and stay in an upright position for the remainder of the day. Patients were reexamined 2 and 4 weeks after the treatment to determine the efficacy of RM.

\section{Results}

Nineteen of the 179 studied patients with MTBI (10.6\%), comprising 8 female and 11 male patients (mean age: $40.4 \pm 10.6$ years, ranging from 16 to 57 years), complained about attacks of vertigo elicited by positional changes or physical exertion which were relieved by rest. All complained about other sensations of persistent dizziness such as light-headedness, floating and drunkenness. None complained of vertigo before the trauma or had any history of the pre-existing inner ear disease that can cause BPPV. In 11 patients, the head trauma was the consequence of a motor vehicle accident, 4 were due to fighting, 2 due to accidents at work, 1 at home, and 1 at a sporting event. The diagnosis of BPPV was confirmed in 9 of these 19 (47.4\%) patients: in 8 cases by a positive Dix-Hallpike test (posterior canal BPPV) and in 1 by the roll test (lateral canal BPPV). In 3 patients, BPPV was bilateral: both ears were found to be simultaneously positive in the Dix-Hallpike test (Table 1).

In 10 out of 179 studied cases (5.6\%), BPPV was diagnosed based on a medical history, which recorded a regular occurrence of vertigo episodes provoked by changes in head position relative to gravity. These observations indicated a high probability of BPPV; 8 of these patients presented posterior BPPV and 2 presented lateral BPPV. No symptoms were observed during the examination. For those with dizziness, the Brandt-Daroff exercises and the roll test were recommended. ${ }^{7,8}$ Videonystagmography compensated unilateral weakness was confirmed in only 1 patient. Eight patients with posterior BPPV confirmed by the DixHallpike test were immediately treated by a single Epley maneuver, while 1 patient with lateral BPPV was treated

Table 1. Clinical data: gender, age and clinical findings in patients $(n=9)$ with post-traumatic BPPV confirmed by maneuvers

\begin{tabular}{|l|c|c|c|}
\hline $\begin{array}{l}\text { Patient No., sex } \\
\text { and age [years] }\end{array}$ & $\begin{array}{c}\text { Semicircular } \\
\text { canal(s) }\end{array}$ & $\begin{array}{c}\text { Repositioning } \\
\text { maneuver }\end{array}$ & Recurrence \\
\hline 1. F, 38 & RP + LP & Epley & $1 \times R P$ \\
\hline 2. F, 28 & RP & Epley & - \\
\hline 3. M,60 & LP + LH & Epley + roll & $2 \times L P$ \\
\hline 4. M, 27 & RP & Epley & - \\
\hline 5. M, 57 & LP + RP & Epley & $1 \times R P$ \\
\hline 6. F,66 & LP & Epley & - \\
\hline 7. F, 62 & RP & Epley & $1 \times R P$ \\
\hline 8. M, 20 & RP & Epley & - \\
\hline 9. M, 23 & LH & roll & - \\
\hline
\end{tabular}

BPPV - benign paroxysmal positional vertigo; $\mathrm{F}$ - female; $\mathrm{M}$ - male; $\mathrm{RP}$ - right posterior; LP - left posterior; $\mathrm{RH}$ - right horizontal; $\mathrm{LH}$ - left horizontal. 
using the barbecue roll. The examination was performed 2 weeks later to determine the efficiency of the treatment. In 4 (44.4\%) patients, the maneuvers were repeated. On the follow-up examination, the patients were not found to have vertigo. All patients were urged to contact the physician if the positional vertigo recurred. In 1 patient, recurrence was confirmed after 2 years by the Dix-Hallpike test. The Epley maneuver was performed with consequent relief of symptoms.

\section{Discussion}

The rapid growth in the occurrence of motor accidents and their ensuing injuries over the last few decades have forced general practitioners, and specialists in laryngology and neurology to consult ever more victims suffering from post-traumatic vertigo or disequilibrium following the accident. In the USA, 1.7 million people sustain a TBI each year, and an analysis of multiorgan injuries found that head injury was confirmed in $72 \%$ of cases. It can be concluded that the head is the region of the human body most susceptible to trauma. In the European Union ("old" Member States), approx. 7.7 million people who have experienced an MTBI have disabilities. ${ }^{1-5}$

Although head trauma is considered a common cause of BPPV, few studies have examined the clinical results and outcome of traumatic BPPV.7,12-14,17,18 In addition, due to the wide inclusion criteria concerning the type of head trauma, its severity, time of examination and analysis used when recruiting patients after head trauma, considerable variation can be seen in the results. Few articles present the outcome of treating traumatic BPPV. ${ }^{8,24}$

In the present study, BPPV was diagnosed in $10.6 \%$ of the patients with vertigo and disequilibrium after head trauma. The incidence, duration, frequency, and intensity of BPPV symptoms vary, perhaps due to the frequent occurrence of spontaneous recovery. Katsarkas identified post-traumatic etiology in $6.2 \%$ of 1644 BPPV patients. ${ }^{12}$ Baloh et al. noted the presence of BPPV in 240 patients, and attributed this to traumatic head injury in $17 \%{ }^{18}$ Ernst et al. diagnosed BPPV in $50 \%$ of patients on the $1^{\text {st }}$ day after head trauma, but only $14 \%$ were confirmed using the Dix-Hallpike test. ${ }^{17}$ Hoffer et al. identified BPPV in $29 \%$ of patients complaining of moderate and severe head trauma. ${ }^{22}$ Such variability in BPPV diagnosis could be attributable to differences in inclusion criteria, variability in the patients who need medical care, and because this type of vertigo may appear at different times following injury, from 1 day to a few months.

As these studies do not include data concerning moderate injuries, for example those occurring in the home, any inferences regarding the "true" incidence of BPPV should therefore be made with caution. ${ }^{14,28}$ Our results confirm those of Katsarkas, who note that the prevalence of post-traumatic BPPV was independent of sex. ${ }^{12}$ Also, our findings indicate the presence of bilateral BPPV in 33\% of all BPPV cases, compared to $14.3 \%$ according to Katsarkas, $40 \%$ by Motin et al., $55 \%$ by Liu and only $6.3 \%$ by Marzo et al.9,12,14,19

The present study diagnoses BPPV in patients from 1 to 2 months after injury, but this criterion differs in other studies: Liu evaluated patients after 3 months, Ernst et al. from 3 weeks to 3 months, and Motin et al. examined the subjects over a period of 18 months following head trauma. ${ }^{9,17,19}$ In addition, considerable variation in diagnostic procedures and treatment options regarding the management of BPPV can be seen between different medical specialties and disciplines. ${ }^{8}$ Repeated testing is recommended on separate occasions to avoid missing a diagnosis. Failure to diagnose BPPV may lead to costly and potentially unnecessary diagnostic testing and/or radiological imaging. ${ }^{8,11}$

In 8 of our 19 patients (42.1\%), the diagnosis of BPPV was confirmed by a positive Dix-Hallpike test. In the study including 100 patients with vertigo or BPPV after head trauma, Davies and Luxon established a diagnosis of anamnesis in $61 \%$ of patients but only $25 \%$ were confirmed by a positive Dix-Hallpike test result. ${ }^{20}$ Nguyen-Huynh reported that even in a specialty clinic, $11 \%$ of patients did not give a positive Dix-Hallpike result on initial examination. ${ }^{11}$ The Dix-Hallpike test is considered the gold standard for the diagnosis of posterior canal BPPV and it is the most common diagnostic criterion required in clinical trials. ${ }^{10,11}$ Based on a review of published literature, some authors estimate the sensitivity of the Dix-Hallpike test to range from $48 \%$ to $88 \%$, but with poor specificity. ${ }^{27,29}$ Guidelines specify that a positive test result is sufficient for diagnosis of BPPV but a negative test result should not rule out BPPV completely. ${ }^{8,11}$

In the current study, $44.4 \%$ of patients with BPPV reported complete symptom resolution after a single repositioning maneuver. The other patients reported resolution after repeated procedures performed over the next 2 weeks, followed by re-examination. During a 2-year follow-up period, only 1 patient has reported a recurrent attack.

Motin et al. reported the resolution of vertigo after the $1^{\text {st }}$ treatment in $40 \%$ of patients and Liu in 39\% of patients. ${ }^{9,19}$ These results are not as good as those observed for idiopathic BPPV. ${ }^{29,30}$ Some authors suggest that trauma may lead to a loss of otolith quantity by microscopic hemorrhage in the inner ear or possibly a wide range of vestibular pathologies, such as blood and tissue degeneration with slight loosening. Otoconia are detached by trauma and microscopic hemorrhages, or by tissues shearing. Experimental head injury in guinea pigs showed a disarrangement of the vestibular system with lithic, exfoliate vacuolization of the sensory epithelia, and exfoliated membranes in the utricular and saccular maculae with otolith separation from the saccular maculae.

Laryngologists, neurologists and primary care physicians are involved in the identification, monitoring and management of patients with BPPV. Establishing a diagnosis 
of BPPV is important since it is treated by relatively simple physical maneuvers without the need for additional investigations or drug therapy. Some authors report that early education is beneficial for recovery in patients after MTBI. ${ }^{5}$ Misunderstanding the reason of dizziness and vertigo in BPPV may influence the emotional state of the patient. In the present study, patients were informed of the treatment options, as well as the risks associated with symptomatic BPPV, including those of temporary dizziness or disorientation, and the possible recurrence of BPPV.

\section{Conclusion}

Despite the fact that the occurrence of BPPV in patients with MTBI is not frequent, BPPV should be diagnosed and treated successfully without any medication, no surgery and no special equipment.

\section{References}

1. Donovan J, Cancelliere C, Cassidy JD. Summary of the findings of the International Collaboration on Mild Traumatic Brain Injury Prognosis. Chiropr Man Therap. 2014;22(1):38. doi: 10.1186/s12998-014-0038-3

2. Roozenbeek B, Mass A, Menon D. Changing patterns in the epidemiology of traumatic brain injury. Nat Rev Neurol. 2013;9(4):231-236.

3. Thurman DJ, Alverson C, Dunn KA, Guerrero J, Sniezek JE. Traumatic brain injury in the United States: A public health perspective. $J$ Head Trauma Rehabil. 1999;14(6):602-615.

4. Cassidy JD, Cancelliere C, Carroll LJ, et al. Systematic review of selfreported prognosis in adults after mild traumatic brain injury: Results of the International Collaboration on Mild Traumatic Brain Injury Prognosis. Arch Phys Med Rehabil. 2014;95:(Suppl 2):132-151.

5. Nygren-de Boussard C, Holm LW, Cancelliere C, et al. Nonsurgical interventions after mild traumatic brain injury: A systematic review. Results of the International Collaboration on Mild Traumatic Brain Injury Prognosis. Arch Phys Med Rehabil. 2014;95:(Suppl 3):257-264.

6. Ruff RM, Iverson GL, Barth JT, et al. NAN Policy and Planning Committee. Recommendations for diagnosing a mild traumatic brain injury: A National Academy of Neuropsychology education paper. Arch Clin Neuropsychol. 2009;24(1):3-10.

7. Brandt T. Traumatic vertigo. In: Brandt T, ed. Vertigo: Its Multisensory Syndromes. $2^{\text {nd }}$ ed. London, UK: Springer-Verlag; 2000;343-354.

8. Bhattacharyya N, Baugh RF, Orvidas L, et al. Clinical practice guideline: Benign paroxysmal positional vertigo. Otolaryngol Head Neck Surg. 2008;139(5 Suppl 4):47-81.

9. Motin M, Keren O, Groswasser Z, Gordon CR. Benign paroxysmal positional vertigo as the cause of dizziness in patients after severe traumatic brain injury: Diagnosis and treatment. Brain Inj. 2005;19(9): 693-697.
10. von Brevern $M$, Radtke $A$, Lezius F, et al. Epidemiology of benign paroxysmal positional vertigo: A population based study. J Neurol Neurosurg Psychiatry. 2007;78(7):710-715.

11. Nguyen-Huynh AT. Evidence-based practice: Management of vertigo. Otolaryngol Clin North Am. 2012;45(5):925-940.

12. Katsarkas A. Benign paroxysmal positional vertigo (BPPV): Idiopathic versus post-traumatic. Acta Otolaryngol. 1999;119(7):745-749.

13. Gordon CR, Levite R, Joffe V, Gadoth N. Is posttraumatic benign paroxysmal positional vertigo different from the idiopathic form? Arch Neurol. 2004;61(10):1590-1593.

14. Marzo SJ, Leonetti JP, Raffin MJ, et al. Diagnosis and management of post-traumatic vertigo. Laryngoscope. 2004;114(10):1720-1723.

15. Hoffer ME, Balough BJ, Gottshall KR. Posttraumatic balance disorders. Int Tinnitus J. 2007;13(1):69-72.

16 Gottshall K, Drake A, Gray N, McDonald E, Hoffer ME. Objective vestibular tests as outcome measures in head injury patients. Laryngoscope. 2003;113(10):1746-1750.

17. Ernst A, Basta D, Seidl RO, Todt I, Scherer H, Clarke A. Management of posttraumatic vertigo. Otolaryngol Head Neck Surg. 2005;132(4):554-558.

18. Baloh RW, Honrubia V, Jacobson K. Benign positional vertigo: Clinical and oculographic features in 240 cases. Neurology. 1987;37(3):371-378.

19. Liu H. Presentation and outcome of post-traumatic benign paroxysmal positional vertigo. Acta Otolaryngol. 2012;132(8):803-806.

20. Davies RA, Luxon LM. Dizziness following head injury: A neuro-otological study. J Neurol. 1995;242(4):222-230.

21. Gurr B, Moffat N. Psychological consequences of vertigo and the effectiveness of vestibular rehabilitation for brain injury patients. Brain Inj. 2001;15(5):387-400.

22. Hoffer ME, Gottshall KR, Balough BJ, Wester D. Characterizing and treatment dizziness after mild head trauma. Otol Neurotol. 2004;25 (2);135-138.

23. Fife TD, Iverson DJ, Lempert T, et al. Practice parameter: Therapies for benign paroxysmal positional vertigo (an evidence-based review): Report of the Quality Standards Subcommittee of the American Academy of Neurology. Neurology. 2008;70(22):2067-2074.

24. Liu Y, Wang W, Zhang AB, Bai X, Zhang S. Epley and Semont maneuvers for posterior canal benign paroxysmal positional vertigo: A network meta-analysis. Laryngoscope. 2016;126(4):951-955. doi: 10.1002/ lary. 25688

25. Epley J. The canalith repositioning procedure: For treatment of benign paroxysmal positional vertigo. Otolaryngol Head Neck Surg. 1992;107(3):399-404.

26. von Brevern M, Bertholon P, Brandt T, et al. Benign paroxysmal positional vertigo: Diagnostic criteria. J Vest Res. 2015;25(3-4):105-117.

27. Kim JS, Zee DS. Benign paroxysmal positional vertigo. N Engl J Med. 2014;370(12):1138-1147.

28. Cohen HS, Kimball KT, Stewart MG. Benign paroxysmal positional vertigo and comorbid conditions. ORL J Otorhinolaryngol Relat Spec. 2004;66(1):11-15.

29. Helminski JO, Zee DS, Janssen I. Effectiveness of particle repositioning maneuvers in the treatment of benign paroxysmal vertigo: A systematic review. Phys Ther. 2010;90(5):663-678.

30. Steenerson RL, Cronin GW, Marbach PM. Effectiveness of treatment techniques in 923 cases of benign paroxysmal positional vertigo. Laryngoscope. 2005;115(2):226-231. 